EPJ manuscript No.

(will be inserted by the editor)

\title{
Size-dependent effects in solutions of small metal nanoparticles
}

\author{
Vitaliy N. Pustovit ${ }^{1,2}$, Tigran V. Shahbazyan ${ }^{2}$ and Leonid G. Grechko ${ }^{1}$ \\ 1 Laboratory of Surface Physics, Institute of Surface Chemistry, Kyiv 03164, Ukraine \\ 2 Department of Physics, Jackson State University, Jackson, MS 39217, USA
}

Received: date / Revised version: date

\begin{abstract}
A new theoretical approach for the calculation of optical properties of complex solutions is proposed. It is based on a dielectric matrix $\varepsilon_{m}$ with included small metallic inclusions (less than $3 \mathrm{~nm}$ ) of spherical shape. We take into account the mutual interactions between the inclusions and the quantum finite-size effects. On the basis of the effective medium model, TDLDA and Kohn-Sham theories, some analytical expressions for the effective dielectric permittivity of the solution are obtained.
\end{abstract}

PACS. PACS-key 78.67.Bf - PACS-key 73.20.Mf

\section{Introduction}

The optical properties of the solutions with small metal particles have attracted much attention in recent years. A number of theories have been proposed for describing the anomalous far-infrared absorption enhancement in this kind of systems [1]. Most authors agree that a direct account of the interaction between particles in such systems has a significant influence on the behavior of the absorption spectra of these systems [2]. In many previous works mutual interactions between particles were taken into account [2]-8], though until now the influence of quantumsize effects for interacting small metal nanoparticlers on the absorption spectra remains uninvestigated. In this pa- per, we show influence of the quantum-size effects on the optical properties of the solutions (matrix disperse systems) based on dielectric matrix and embedded in small metallic inclusions (less $3 \mathrm{~nm}$ ) of spherical shape. We demonstrate that level of mutual interactions between metallic inclusions is increased, especially for the small size nanoparticles where quantum-size effects became most pronounced [9. The underlying mechanism of enhancement is related to the difference in the density profiles of $s p$ band and $d$-band electrons near the nanoparticle boundary. Specifically, the localized $d$-electrons are mainly confined within nanoparticle classical volume while the wavefunctions of delocalized sp-electrons extend outside of it. 
Please give a shorter version with: \authorrunning and \titlerunning prior to \maketitle

This spillover leads to a larger effective radius for $s p$ electrons [10] and, thus, to the existence of a surface layer with diminished $d$-electron population. 1912 As a result, in the surface layer, the screening of $s p$-electrons by $d$-band electron background is reduced, leading to the enhancement of the local field and correspondingly to the mutual interactions between neighboring metallic particle.

The paper is organized as follows. In Section 2 we develop model of extended Maxwell-Garnett approximation [13] which will includes all mutual multipolar interactions between neighbor metal inclusions [5]. In Section $A$ we calculate absorption spectra for the solution of Au nanoparticles. Discussion of our numerical results is presented in the same Section. In Appendix we propose original way for derivation of quantum multipolar polarizability of metal nanoparticle. Section 4 concludes the paper.

\section{Extended Maxwell-Garnett approximation}

Consider a system of $\mathrm{N}$ non-overlapping spherical metallic particles randomly distributed in the background media and interacting with each other and with an incident plane monochromatic wave, whose wavelength $\lambda$ is assumed to be much larger than the average size of the particles in the cluster (i.e., electrostatic approximation) [14]. The particles have approximately the same radius $R$ and embedded in a dielectric continuous matrix with dielectric constant $\varepsilon_{m}$. Due to the influence of the external field as well as the interaction between particles in the solution, all particles in the system will obtain some polarization. The total effective dipole moment of particle $i$, accounting mutual neighboring many-particle interactions, can be expressed as

$$
\mathbf{p}_{i}(1,2 \ldots N)=\mathbf{p}_{i}^{(0)}+\sum_{j \neq i}^{N} \mathbf{p}_{i j}+\ldots,
$$

where is a dipole moment of an isolated particle $i$ induced by the external field $\mathbf{E}_{\mathbf{0}}$. The second term in the expansion (1) is defined by

$$
\mathbf{p}_{i j}=\mathbf{p}(i, j)-\mathbf{p}_{i}^{(0)}
$$

where $\mathbf{p}(i, j)$ is the dipole moment induced in particle $i$ in the presence of a second particle $j$. The second term in expansion (1-2) defines pair interactions between particles in the given mixture, the third three- particle interactions, and so on. We have restricted ourselves to considering the case of pair interactions between particles, because the inclusion of the third term will render the system mathematically hard to treat. As it is follow from the Maxwell equations, in the electrostatic approximation the average Lorentz field is connected with average polarization in the system through the Green function equation

$$
\langle\mathbf{F}(r)\rangle=\mathbf{E}_{0}+\int G\left(\mathbf{r}-\mathbf{r}^{\prime}\right)\left\langle\mathbf{P}\left(r^{\prime}\right)\right\rangle d r^{\prime}
$$

From the other side, the averaged macroscopic polarization is linked with averaged Lorentz field in the solution as

$$
\langle\mathbf{P}\rangle=\frac{3}{4 \pi} \frac{\hat{\varepsilon}-\varepsilon_{m}}{\hat{\varepsilon}+2 \varepsilon_{m}}\langle\mathbf{F}\rangle
$$

where $\hat{\varepsilon}$ is a effective dielectric permittivity of the solution. Since we have restricted ourselves with first two terms of expansion (1), the averaged macroscopic polarization of the solution becomes

$$
\langle\mathbf{P}(1,2 \ldots N\rangle)=\mathbf{p}_{i}^{(0)}+\frac{1}{V} \sum_{i \neq j} \mathbf{p}_{i, j}\left(\mathbf{r}_{i}, \mathbf{r}_{j}\right) \Phi\left(\mathbf{r}_{i}, \mathbf{r}_{j}\right) d r_{j}
$$




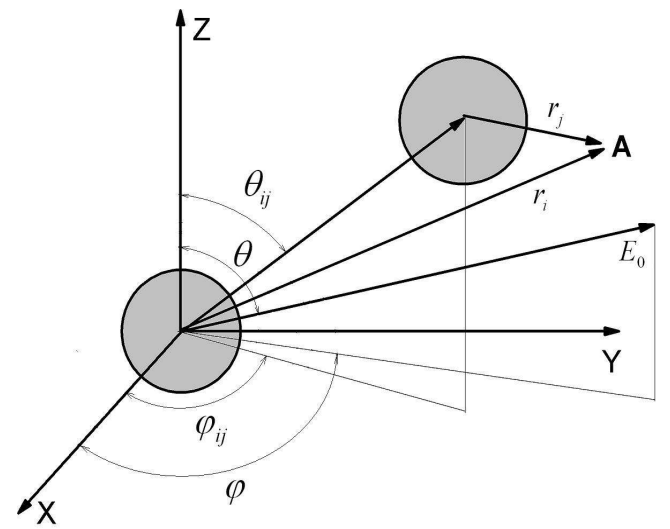

Fig. 1. Two metallic nanoparticles in the external field $\mathbf{E}_{0}$,

where $V$ is a total system volume and $\Phi\left(\mathbf{r}_{i}, \mathbf{r}_{j}\right)$ is a pair correlation function of the particle distribution in the solution.

In order to find expression for the pair dipole polarization $\mathbf{p}_{i, j}$ lets us solve two particle problem in the electrostatic approximation.

First, we denote by $\mathbf{r}_{i}$ the center of the sphere $i$ and by $r$ an arbitrary point in the medium. The potential inside sphere $i$ can be expressed in the form:

$$
\Psi_{i n}^{(i)}=-\mathbf{E}_{\mathbf{0}} \sum_{l m} C_{l m}^{(i)}\left|r-r_{i}\right|^{l} Y_{l m}\left(r \hat{-} r_{i}\right),
$$

where $r \hat{-} r_{i}$ is the unit vector along the direction $r-r_{i}$ and $Y_{l m}\left(r \hat{-} r_{i}\right)$ are the scalar spherical harmonics (SSH) so $l$ is the polar order. The potential outside sphere $i$ can be expressed in the form:

$$
\begin{array}{r}
\Psi_{\text {out }}^{(i)}=-\mathbf{E}_{\mathbf{0}} \sum_{l m} d_{l m}\left|r-r_{i}\right|^{l} Y_{l m}\left(r \hat{-} r_{i}\right) \\
-\mathbf{E}_{\mathbf{0}} \sum_{l m} B_{l m}^{(i)}\left|r-r_{i}\right|^{-l-1} Y_{l m}\left(r \hat{-} r_{i}\right) \\
-\mathbf{E}_{\mathbf{0}} \sum_{j \neq i} \sum_{l m} B_{l m}^{(j)}\left|r-r_{j}\right|^{-l-1} Y_{l m}\left(r \hat{-} r_{j}\right),
\end{array}
$$

where the first term is the potential of the external field, the second term is the potential created by the particle $i$ at the point $\mathbf{r}$, and the third term is the potential created by the rest of the particles of the solution at this point. Breaking up the potential inside the sphere into components in the two regions and applying standard boundary conditions at the three boundaries, plus use of

$$
\begin{aligned}
d_{l m}=-\delta_{l 1} \sqrt{\frac{2 \pi}{3}}\left\{\sqrt{2} \delta_{m 0} \mathbf{u}_{z}\right. & +i\left[\delta_{m 1}+\delta_{m-1}\right] \mathbf{u}_{y} \\
& \left.+\left[\delta_{m-1}-\delta_{m 1}\right] \mathbf{u}_{x}\right\}
\end{aligned}
$$

where $\mathbf{u}=\mathbf{E}_{\mathbf{0}} / E_{0}$, leads to the coupled set of equations

$$
\sum_{l^{\prime}=1}^{\infty} T_{l l^{\prime}}^{(m)} X_{l^{\prime} m}=\delta_{l 1} ; \quad l=1,2 \ldots ; \quad m=-l, \ldots l,
$$

where

$$
T_{l l^{\prime}}^{(m)}=\frac{R^{2 l+1}}{\alpha_{l}} \delta_{l l^{\prime}}-(-1)^{m}\left(R / r_{i j}\right)^{l+l^{\prime}+1}\left(\begin{array}{c}
l+l^{\prime} \\
l+m
\end{array}\right)
$$

where $R$ is a radius of nanoparticle, and $X_{l m}=B_{l m} R^{-(l+2)}$ with symbol

$$
\left(\begin{array}{c}
l \\
m
\end{array}\right)=\frac{l !}{m !(l-m) !}
$$

Following the classical approach for polarizability of the single spherical particle we should apply an expression which can be easily derived from the Mie theory [14] $\alpha_{l}=$ $R^{2 l+1} l\left(\varepsilon-\varepsilon_{m}\right) /\left(l \varepsilon+(l+1) \varepsilon_{m}\right)$. Although, if we consider small size nanoparticles (less $2 \mathrm{~nm}$ ), that might be necessary to take into account finite-size effects [9]. The role of interband screening and $s p$ band spillover on the boundary of small particle will make a significant influence on the general multipolar polarizability. The quantum multipolar polarizabily in Eq.(10) can be defined in terms of 
induced density of electrons $9.6 n=\delta n_{s}+\delta n_{d}+\delta n_{m}$ due to influence of interband screening and $s p$ band spillover as $\alpha_{l}^{q}=\alpha_{l}^{s}+\alpha_{l}^{d}$ where

$$
\begin{gathered}
\alpha_{l}^{q}=A_{l} R^{2 l+1}=\int_{0}^{\infty} d r^{\prime} r^{\prime l+2} \delta n^{(l)}\left(r^{\prime}\right), \\
\alpha_{l}^{d}=\frac{l R^{2 l+1}}{\eta}\left[\lambda_{d} a^{2 l+1}\left(1-\lambda_{m}\right)-\lambda_{m}\right], \\
\alpha_{l}^{s}=\int_{0}^{\infty} d r^{\prime} r^{l+2} \delta n_{s}^{(l)}\left(r^{\prime}\right) \\
-\frac{l(2 l+1) R_{d}^{l+1} \lambda_{d}}{4 \pi \eta} \int_{0}^{\infty} d r^{\prime} r^{\prime 2} \delta n_{s}^{(l)}\left(r^{\prime}\right)\left[B_{l}\left(R_{d}, r^{\prime}\right)\right. \\
\left.-a^{l}(l+1) \lambda_{m} B_{l}\left(R, r^{\prime}\right)\right] \\
+\frac{l(2 l+1) R^{l+1} \lambda_{m}}{4 \pi \eta} \int_{0}^{\infty} d r^{\prime} r^{\prime 2} \delta n_{s}^{(l)}\left(r^{\prime}\right)\left[B_{l}\left(R, r^{\prime}\right)\right. \\
\left.-l a^{l+1} \lambda_{d} B_{l}\left(R_{d}, r^{\prime}\right)\right],
\end{gathered}
$$

with coefficients given in Appendix A

Finally, from the boundary equations plus Eqs. (5-9) we obtain the expression for pair dipole moment

$$
\mathbf{p}(i, j)=-\mathbf{E}_{0} \sum_{m=1}^{1} B_{1 m}^{(i)} Y_{1 m}\left(\mathbf{r}_{i}-\mathbf{r}_{j}\right)
$$

We can now derive an expression for $\mathbf{p}_{i, j}$ as

$$
\mathbf{p}_{i, j}=R^{3}\left[X_{10} n_{z} u_{z}+X_{11}\left(n_{x} u_{x}+n_{y} u_{y}\right)\right] \mathbf{E}_{0}
$$

where $\mathbf{n}=\mathbf{r}_{\mathbf{i j}} / r_{i j}$ and $r_{i j}=\left|r_{i}-r_{j}\right|$. Here coefficients $X_{10}$ and $X_{11}$ are obtained from the coupled system of equations Eq.(9). By selecting position of two particles along the axis $Z$ and parallel to external field $\mathbf{E}_{\mathbf{0}}$, we can investigate dependence of the pair dipole interaction magnitude on the distance between two particles (see Fig 2). Starting with small size of particle $92 e$ or $R=0.7 \mathrm{~nm}$ the maximum contribution to the absorption spectra will bring the particles located on relatively far distances from each other, i.e. 10, 20 and $40 \mathrm{~nm}$. That conclusion lets us to assume

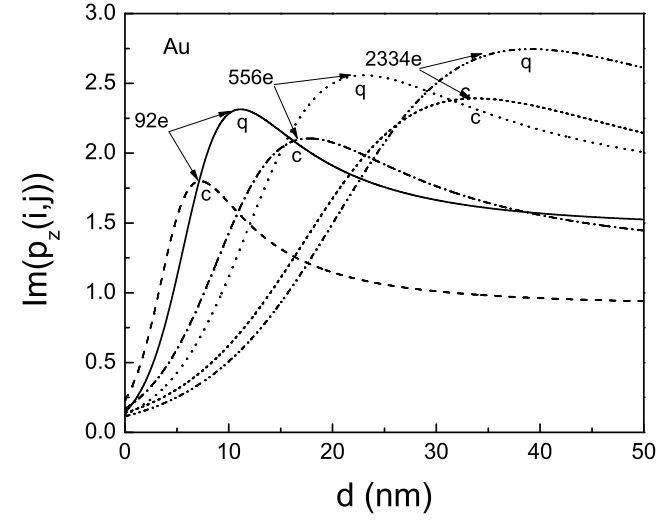

Fig. 2. Image part of the pair dipole interaction given in Eq.(14) in $Z$ direction vs distance between two particles. The letters "c" and "q" defines application of the classical and TDLDA methods correspondingly for particles with 92e, $R=0.7 \mathrm{~nm} ; 556 \mathrm{e}, R=1.3 \mathrm{~nm}$ and $2334 \mathrm{e}, R=2 \mathrm{~nm}$.

that further in our calculations we can restrict ourselves only with the dipole $l=1$ interactions between particles, neglecting with higher orders multipoles, which are important for the close distances. After performing integration over distance between each pair of particle in Eq.(5) with pair correlation function $\Phi\left(r_{i j}\right)$ and taking into account expression for the Lorentz field in Eq.(4), we get a final expression for calculation of the effective dielectric permittivity of the solution with account of pair multipole interaction between metallic nanoparticles [5] - [6]

$$
\begin{aligned}
& \frac{\hat{\varepsilon}+2 \varepsilon_{m}}{\hat{\varepsilon}-\varepsilon_{m}}=\frac{1}{f A_{1}} \\
& -\frac{1}{A_{1}^{2} R^{6}} \int_{0}^{\infty} r_{i j}^{2} \Phi\left(r_{i j}\right)\left[\beta^{\|}\left(r_{i j}\right)+2 \beta^{\perp}\left(r_{i j}\right)\right] d r_{i j},
\end{aligned}
$$

where parameters

$$
\beta^{\|}=X_{11}-A_{1}, \quad \beta^{\perp}=X_{10}-A_{1},
$$

where $A_{1}$ is given by the $l=1$ term in Eq.(12) and $f=\frac{4 \pi}{3} R^{3} n$ is a volume fraction of metallic particles in so- 
lution with concentration $n$. The second term on the right side of Eq.(15) is a contribution of the pair interaction between particles. Omitting this term, Eq.(15) is easily reduced to the simple traditional form of the MaxwellGarnett approximation [13].

\section{Numerical results and discussion}

Below we present results of our calculations for Au nanoparticles with the same radius ranging from 0.7 to $2 \mathrm{~nm}$ in a medium with dielectric constant $\varepsilon_{m}=1.77$. The surface plasmon (SP) resonance is positioned at $2.35 \mathrm{Ev}$, far away from the interband transitions for gold [18. Further we will consider absorption properties of the given solution with metallic inclusions in the SP frequency region. The volume fraction of nanoparticle inclusions in the solution is $f=0.1$ and we used the simplest approximation for the two particle distribution function

$$
\Phi\left(r_{i j}\right)=\left\{\begin{array}{cc}
1 & r_{i j}>3 R \\
0 & r_{i j}<3 R
\end{array},\right.
$$

where minimal possible distance between two particles is selected to be at least more than one radius or $3 R$ between particle centers.

To ensure spherical symmetry, only closed-shell magic numbers $N$ of electrons 92,556 and 2334 were used [17]. For such sizes, the Au band-structure remains intact. The ground-state energy spectrum and wave functions were obtained by solving the Kohn-Sham equations for the jellium model [19] with the Gunnarsson-Lundqvist exchangecorrelation potential [20. These results were used as input in the numerical solution of TDLDA system A(13).

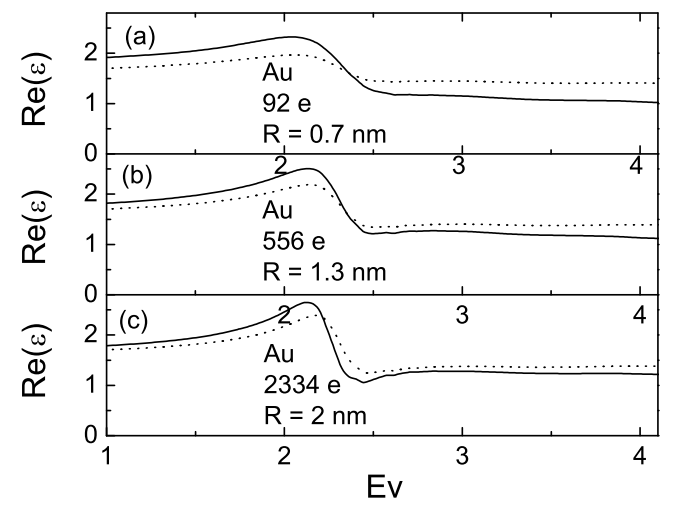

Fig. 3. Calculated real part of the medium effective dielectric constant in dipole approximation for solution with $\mathrm{Au}$ nanoparticles with 92, 556 and 2334 electrons at volume fraction $f=0.1$. The solid line corresponds to the calculations based on TDLDA method, and the dotted corresponds to classical approach.

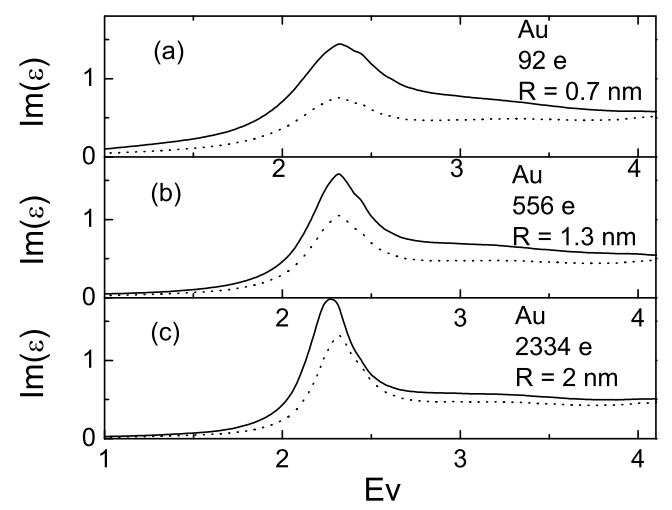

Fig. 4. Calculated image part of the medium effective dielectric constant in dipole approximation for solution with $\mathrm{Au}$ nanoparticles with 92, 556 and 2334 electrons at volume fraction $f=0.1$. The solid line corresponds to the calculations based on TDLDA method, and the dotted corresponds to classical approach.

The effective radius of nanoparticles was taken in form $R=r_{s} N^{1 / 3}$, where parameter $r_{s}$, defined as radius in atomic units, is equal to 3 a.u. for Au. On Fig 3 and Fig 4 are shown calculated real and image parts of ef- 
fective dielectric permittivity of given solution obtained from Eq. (15). Here we can compare absorption $(\operatorname{Im}(\hat{\varepsilon}))$ computed with use of TDLDA quantum polarizability $\alpha_{l}^{q}$ (layer with sp-free electrons is included with parameter $\Delta=1$, see derivations in $[9$ ) with absorption computed classically with polarizability $\alpha_{l}$. For all three cases (a)(c) on Fig 3 and Fig 4 results based on TDLDA display higher level of absorption in the system, especially for the solution with small particles (like 92 e). In such case of small particle size the difference between two magnitudes can reach almost 2 times and significantly reduces with increase of particle radius. This fact allows us to predict that in the solutions with small particles the level of absorption can be higher than it was expected before based on the classical calculations.

\section{Conclusions}

We proposed a theoretical method for calculation of optical properties of the complex solutions based on dielectric matrix $\varepsilon_{m}$ with included in small metallic inclusions of spherical shape with account of the dipole interaction between inclusions and quantum finite-size effects. We shown that optical properties of given solutions are very much dependent on the proper account of microscopic effects, in particular, electron screening in the surface layer of nanoparticles. The exit of delocalized s- electrons out of the classical boundary leads to the additional local field enhancement and as a consequence to enhancement of the surface plasmon of nanoparticle. That correspondingly makes influence on the character of the dipole interaction between nanoparticles in solution and on the optical properties of this solution.

\section{A Derivation of quantum multipolar}

\section{polarizability}

The full self-consistent potential $\Psi(\mathbf{r})=\psi(\mathbf{r})+\psi_{0}(\mathbf{r})$ satisfies Poisson equation

$$
\Psi(\mathbf{r})=\psi_{0}(\mathbf{r})+e^{2} \int d^{3} r^{\prime} \frac{\delta N\left(\mathbf{r}^{\prime}\right)}{\left|\mathbf{r}-\mathbf{r}^{\prime}\right|},
$$

where $\psi_{0}(\mathbf{r})=-e \mathbf{E}_{0} \dot{\mathbf{r}}$ is potential of the external electric field, the induced density is comprised of $s p$-band, $d$-band and medium contributions, $\delta N(\mathbf{r})=\delta N_{s}(\mathbf{r})+\delta N_{d}(\mathbf{r})+$ $\delta N_{m}(\mathbf{r})$. The induced density of $s p$-band electrons is determined from TDLDA equation presented below and contributions from d-electrons $\delta N_{d}(\mathbf{r})$ and medium $\delta N_{m}(\mathbf{r})$ are back to $\Psi(\mathbf{r})$ as

$$
\begin{aligned}
\delta N_{d}(\mathbf{r}) & =\nabla\left[\chi_{d}(r) \nabla \Psi(\mathbf{r})\right], \\
\delta N_{m}(\mathbf{r}) & =\nabla\left[\chi_{m}(r) \nabla \Psi(\mathbf{r})\right],
\end{aligned}
$$

where $\chi_{d}(r)=\frac{\epsilon_{d}-1}{4 \pi} \theta\left(R_{d}-r\right)$ is the interband susceptibility with the step function enforcing the boundary conditions and, correspondingly, $\chi_{m}(r)=\frac{\epsilon_{m}-1}{4 \pi} \theta(r-R)$ is the susceptibility of surrounding medium. The induced density of $s p$-band electrons is given as

$$
\delta N_{s}(\mathbf{r})=\frac{4 \pi \delta n_{s}(r)}{(2 l+1) r^{2 l+1}}
$$

Integrating by parts, Eq. (18) takes the form

$$
\begin{array}{r}
\epsilon(r) \Psi(\mathbf{r})=\psi_{0}(\mathbf{r})+e^{2} \int d^{3} r^{\prime} \frac{\delta N_{s}\left(\mathbf{r}^{\prime}\right)}{\left|\mathbf{r}-\mathbf{r}^{\prime}\right|} \\
+\frac{\epsilon_{d}-1}{4 \pi} \int d^{3} r^{\prime} \nabla^{\prime} \frac{1}{\left|\mathbf{r}-\mathbf{r}^{\prime}\right|} \cdot \nabla^{\prime} \theta\left(R_{d}-r\right) \Psi\left(\mathbf{r}^{\prime}\right)
\end{array}
$$


Please give a shorter version with: \authorrunning and \titlerunning prior to \maketitle

$$
+\frac{\epsilon_{m}-1}{4 \pi} \int d^{3} r^{\prime} \nabla^{\prime} \frac{1}{\left|\mathbf{r}-\mathbf{r}^{\prime}\right|} \cdot \nabla^{\prime} \theta(r-R) \Psi\left(\mathbf{r}^{\prime}\right)
$$

where $\epsilon(r)=\left(\epsilon_{d}, 1, \epsilon_{m}\right)$ for $r$ in the intervals $[(0, R)$, $\left.\left(R_{d}, R\right),(R, \infty)\right]$, respectively. We expand $\Psi$ and $\delta N_{s}$ in terms of spherical harmonics and obtain,

$$
\begin{array}{r}
\epsilon(r) \psi_{l}(r)=\psi_{0}(\mathbf{r}) \\
+e^{2} \int d r^{\prime} r^{\prime 2} B_{l}\left(r, r^{\prime}\right) \delta N_{s}\left(r^{\prime}\right) \\
-\frac{\epsilon_{d}-1}{4 \pi} R_{d}^{2} \partial_{R_{d}} B_{l}\left(r, R_{d}\right) \psi_{l}\left(R_{d}\right) \\
+\frac{\epsilon_{m}-1}{4 \pi} R^{2} \partial_{R} B_{l}(r, R) \psi_{l}(R),
\end{array}
$$

where

$$
B_{l}\left(r . r^{\prime}\right)=\frac{4 \pi}{2 l+1}\left[\frac{r^{\prime l}}{r^{l+1}} \theta\left(r-r^{\prime}\right)+\frac{r^{l}}{r^{\prime l+1}} \theta\left(r^{\prime}-r\right)\right]
$$

is the multipole term of the radial component of the Coulomb potential. The above equation can be simplified to

$$
\begin{array}{r}
\epsilon(r) \psi_{l}(r)=\bar{\psi}_{l}(r)- \\
-\frac{\epsilon_{d}-1}{2 l+1} \beta_{l}\left(r / R_{d}\right) \psi_{l}\left(R_{d}\right) \\
+\frac{\epsilon_{m}-1}{2 l+1} \beta_{l}(r / R) \psi_{l}(R),
\end{array}
$$

where we introduced a shorthand notation

$$
\bar{\psi}_{l}(r)=\psi_{0}(\mathbf{r})+e^{2} \int d r^{\prime} r^{2} B_{l}\left(r, r^{\prime}\right) \delta N_{s}\left(r^{\prime}\right),
$$

and $\beta_{l}(r / R)=\frac{2 l+1}{4 \pi} R^{2} \partial_{R} B_{l}(R, r)$ is given by

$$
\beta_{l}(x)=l x^{-(l+1)} \theta(x-1)-(l+1) x^{l} \theta(1-x) .
$$

The boundary values of $\psi$ can be obtained by matching $\psi(r)$ at $r=R_{d}, R$

$$
\begin{aligned}
\left(l \epsilon_{d}+l+1\right) \psi_{l}\left(R_{d}\right) & +(l+1) a^{l}\left(\epsilon_{m}-1\right) \psi_{l}(R) \\
& =(2 l+1) \bar{\psi}_{l}\left(R_{d}\right)
\end{aligned}
$$

$$
\begin{gathered}
l\left(\epsilon_{d}-1\right) a^{l+1} \psi_{l}\left(R_{d}\right)+\left(l \epsilon_{m}+\epsilon_{m}+1\right) \psi_{l}(R) \\
=(2 l+1) \bar{\psi}_{l}(R)
\end{gathered}
$$

where $a=R_{d} / R$. Substituting $\psi_{l}\left(R_{d}\right)$ and $\psi_{l}(R)$ back into Eq. (24), we arrive at

$$
\begin{array}{r}
\epsilon(r) \psi_{l}(r)=\bar{\psi}_{l}(r) \\
-\beta_{l}\left(r / R_{d}\right) \frac{\lambda_{d}}{\eta}\left[\bar{\psi}_{l}\left(R_{d}\right)-(l+1) a^{l} \lambda_{m} \bar{\psi}_{l}(R)\right] \\
+\beta_{l}(r / R) \frac{\lambda_{m}}{\eta}\left[\bar{\psi}_{l}(R)-l a^{l+1} \lambda_{d} \bar{\psi}_{l}\left(R_{d}\right)\right],
\end{array}
$$

where the coefficients $\lambda$ are given by

$$
\begin{aligned}
a=R_{d} / R, \quad \lambda_{d} & =\frac{\epsilon_{d}-1}{l \epsilon_{d}+l+1}, \quad \lambda_{m}=\frac{\epsilon_{m}-1}{l \epsilon_{m}+\epsilon_{m}+1} \\
\eta & =1-l(l+1) a^{2 l+1} \lambda_{d} \lambda_{m}
\end{aligned}
$$

Separating out $\delta n_{s}$-dependent contribution, we arrive at TDLDA equation Ref.

$$
\begin{gathered}
\delta n_{s}^{(l)}(r)=\int d r^{\prime} r^{2} \Pi_{s}^{(l)}\left(r, r^{\prime}\right)\left[w_{0}^{(l)}\left(r^{\prime}\right)+\delta w_{0}^{(l)}\left(r^{\prime}\right)\right] \\
+\int d r^{\prime} r^{\prime 2} \Pi_{s}^{(l)}\left(r, r^{\prime}\right)\left[\int d r^{\prime \prime} r^{\prime \prime 2} A\left(r^{\prime}, r^{\prime \prime}\right) \delta n_{s}^{(l)}\left(r^{\prime \prime}\right)\right. \\
\left.+V_{x}^{\prime}\left(r^{\prime}\right) \delta n_{s}^{(l)}\left(r^{\prime}\right)\right]
\end{gathered}
$$

where $\Pi_{s}\left(\mathbf{r}, \mathbf{r}^{\prime}\right)$ is the polarization operator for noninteracting $s p$-electrons, $V_{x}^{\prime}\left[n\left(r^{\prime}\right)\right]$ is the (functional) derivative of the exchange-correlation potential and $n(r)$ is the ground-state electron density. The latter is obtained in a standard way by solving Kohn-Sham equations. Here we define $w_{0}^{(l)}(r)=r^{l} / \epsilon(r)$ and

$$
\begin{aligned}
\delta w_{0}^{(l)}(r) & =\frac{R^{l}}{\epsilon(r)}\left[-\beta_{l}\left(r / R_{d}\right) \lambda_{d} a^{l}\left(1-(l+1) \lambda_{m}\right) / \eta\right. \\
& \left.+\beta_{l}(r / R) \lambda_{m}\left(1-l a^{2 l+1} \lambda_{d}\right) / \eta\right] \\
\delta w_{s}^{(l)}(r) & =\int d r^{\prime} r^{\prime 2} A\left(r, r^{\prime}\right) \delta n_{s}^{(l)}\left(r^{\prime}\right)
\end{aligned}
$$


where kernel $\mathrm{A}\left(\mathrm{r}, \mathrm{r}^{\prime}\right)$ is a renormalized Coulomb potential $\Psi(r)$ due to d-band and medium expressed as

$$
\begin{array}{r}
A\left(r, r^{\prime}\right)=\frac{1}{\epsilon(r)}\left[B_{l}\left(r, r^{\prime}\right)-\beta_{l}\left(r / R_{d}\right) \frac{\lambda_{d}}{\eta}\left[B_{l}\left(R_{d}, r^{\prime}\right)\right.\right. \\
\left.-a^{l}(l+1) \lambda_{m} B_{l}\left(R, r^{\prime}\right)\right]+\beta_{l}(r / R) \frac{\lambda_{m}}{\eta}\left[B_{l}\left(R, r^{\prime}\right)\right. \\
\left.\left.-l a^{l+1} \lambda_{d} B_{l}\left(R_{d}, r^{\prime}\right)\right]\right]
\end{array}
$$

The expression for quantum nanoparticle polarizability $\alpha^{q}=\alpha_{d}+\alpha_{s}$, can be obtained from the asymptotic for $r \gg R$

$$
\begin{aligned}
\delta w_{0}^{(l)}(r) & =\frac{4 \pi}{(2 l+1) \epsilon_{m} r^{l+1}} \alpha_{d}, \\
\delta w_{s}^{(l)}(r) & =\frac{4 \pi}{(2 l+1) \epsilon_{m} r^{l+1}} \alpha_{s},
\end{aligned}
$$

with

$$
\begin{gathered}
\alpha_{d}=\frac{l R^{2 l+1}}{\eta}\left[\lambda_{d} a^{2 l+1}\left(1-\lambda_{m}\right)-\lambda_{m}\right] \\
\alpha_{s}=\int_{0}^{\infty} d r^{\prime} r^{l+2} \delta n_{s}^{(l)}\left(r^{\prime}\right) \\
-\frac{l(2 l+1) R_{d}^{l+1} \lambda_{d}}{4 \pi \eta} \int_{0}^{\infty} d r^{\prime} r^{\prime 2} \delta n_{s}^{(l)}\left(r^{\prime}\right)\left[B_{l}\left(R_{d}, r^{\prime}\right)\right. \\
\left.-a^{l}(l+1) \lambda_{m} B_{l}\left(R, r^{\prime}\right)\right] \\
+\frac{l(2 l+1) R^{l+1} \lambda_{m}}{4 \pi \eta} \int_{0}^{\infty} d r^{\prime} r^{\prime 2} \delta n_{s}^{(l)}\left(r^{\prime}\right)\left[B_{l}\left(R, r^{\prime}\right)\right. \\
\left.-l a^{l+1} \lambda_{d} B_{l}\left(R_{d}, r^{\prime}\right)\right]
\end{gathered}
$$

\section{References}

1. For a review, see the papers in Electrical Transport and Optical Properties of Inhomogeneous Media; Physica A, 241, (Edited by A.M.Dykhne and A.N.Lagerkov, A.K.Sarychev, 1997), 1-452

2. F.Claro and R.Rojas, Phys. Rev. B 43, (1991) 6369.

3. R. Gunther, D. Heinrich, Z. Physik 185, (1965) 345.
4. S. Giordano, Journal of Electrostatics 63, (2005) 1.

5. L.G.Grechko, V.N.Pustovit, K.W.Whites, Appl. Phys. Lett. 76, (2000) 1854 .

6. L.G.Grechko, V.N.Pustovit, S.N.Shostak, Proc.SPIE, 3890, (1999) 381.

7. B. U. Felderhof, G. W. Ford, and E. G. D. Cohen, J. Stat. Phys.28, (1982) 135.

8. B. U. Felderhof and R. B. Jones, Phys. Rev. B 39, (1989) 5669.

9. V.N. Pustovit and T. V. Shahbazyan, Phys. Rev. B 73, (2006) 085408.

10. B. N. J. Persson and E. Zaremba, Phys. Rev. B 31, (1985) 1863.

11. A. Liebsch, Phys. Rev. B 48, (1993) 11317; A. Liebsch and W. L. Schaich, Phys. Rev. B 52, (1995) 14219.

12. A. Liebsch and W. L. Schaich, Phys. Rev. 52, (1995) 14219.

13. C. Maxwell-Garnett, Philos. Trans. R. Soc. London, Ser. A 203 (1904) 385.

14. C. F. Bohren and P. R. Huffman, Absorption and Scattering of Light by Small Particles, (Wiley, New York, 1983)

15. V.N.Pustovit, L.V.Garanina, L.F.Mironuk, S.V.Shostak, Radio Physics and Radio Astronomy, 3 (1998) 441.

16. L.G.Grechko, V.N.Pustovit, V.V.Boiko, Radio Physics and Radio Astronomy, 3 (1998) 245.

17. W. Ekardt, Phys. Rev. B 31, (1985) 6360.

18. Handbook of Optical Constants of Solids, (Ed. E. D. Palik, Academic Press, 1985).

19. A. Liebsch, Phys. Rev. 48, (1993) 11317.

20. O. Gunnarsson and B. Lundqvist, Phys. Rev. B 13, (1976) 4274 . 